VoL. 41 (1990) [503-507]

\title{
ON THE DROP AND WEAK DROP PROPERTIES FOR A BANACH SPACE
}

\author{
J.R. Giles, Brailey Sims and A.C. Yorke
}

\begin{abstract}
Rolewicz' drop property is a modification of a concept underlying Daneä' drop theorem. We characterise the drop property by the upper semicontinuity and compact valued property of the duality mapping for the dual. The characterisation suggests that we define a weak drop property which we show characterises the reflexivity of the space.
\end{abstract}

Consider the Banach space $(X,\|\cdot\|)$ with open unit ball $B(X) \equiv\{x \in X:\|x\|<1\}$ and closed unit ball $\bar{B}(X) \equiv\{x \in X:\|x\| \leq 1\}$. Given $x \notin \bar{B}(X)$, the set $D(x, \bar{B}(X)) \equiv \operatorname{co}\{x, \bar{B}(X)\}$, the convex hull of $x$ and $\bar{B}(X)$, is called the drop generated by $x$. Daneš $[3]$ proved that in any Banach space $(X,\|\cdot\|)$, for every closed set $C$ at positive distance from $\bar{B}(X)$, there exists an $x \in C$ such that $D(x, \bar{B}(x)) \cap C=\{x\}$. Rolewicz [9], modifying the Daneš' drop theorem assumption, said that the norm $\|\cdot\|$ of $X$ has the drop property if for every closed set $C$ disjoint from $\bar{B}(X)$, there exists an $x \in C$ such that $D(x, \bar{B}(X)) \cap C=\{x\}$. He also introduced an associated sequential concept; a sequence $\left\{x_{n}\right\}$ in $X \backslash \bar{B}(X)$ such that $x_{n+1} \in D\left(x_{n}, \bar{B}(X)\right)$ for all $n$, is called a stream.

Rolewicz proved that

(i) the norm $\|\cdot\|$ has the drop property if and only if each stream in $X \backslash \bar{B}(X)$ contains a convergent subsequence, $[9$, Proposition 2, p.29], and

(ii) if the norm $\|\cdot\|$ has the drop property then $X$ is reflexive, [8, Theorem 5, p.34].

To establish reflexivity, Rolewicz used the following technique. Given a subset $E$ of $X$ we define the Kuratowski index of non-compactness of $E$ as the number $\alpha(E) \equiv \inf \{r: E$ is covered by a finite family of open sets with diameter $<r\}$, (see [1]).

Denoting the unit sphere of $X$ by $S(X) \equiv\{x \in X:\|x\|=1\}$, given $f \in S\left(X^{*}\right)$ and $0<\delta<1$, the slice of $\bar{B}(X)$ defined by $f$ and $\delta$ is the subset $S(\bar{B}(X), f, \delta) \equiv$ $\{x \in \bar{B}(X): f(x) \geq 1-\delta\}$. We say that the norm $\|\cdot\|$ has property $(\alpha)$ for $f \in S\left(X^{*}\right)$ if

Received 11 August 1989

Copyright Clearance Centre, Inc. Serial-fee code: 0004-9729/90 \$A2.00+0.00. 
$\left.\lim _{\delta \rightarrow 0} \alpha(S \bar{B}(X)), f, \delta\right)=0$. In this case $\left.\bigcap_{\delta>0} S \bar{B}(X), f, \delta\right)$ is a non-empty compact set and so $f$ attains its norm on $\bar{B}(X)$. He showed [9, Theorem 4, p.33] that if the norm $\|\cdot\|$ has the drop property then the norm $\|\cdot\|$ has property $(\alpha)$ for all $f \in S\left(X^{*}\right)$ and so $X$ is reflexive by James' characterisation of reflexivity [6]. Kutzarova, [7, Corollary 2, p.19] proved that if $X$ is reflexive then $X$ has an equivalent norm with property $(\alpha)$ for all $f \in S\left(X^{*}\right)$.

The duality mapping for $X$ is the set valued mapping $x \mapsto D(x)$ of $S(X)$ into subsets of $S\left(X^{*}\right)$ where $D(x) \equiv\left\{f \in S\left(X^{*}\right): f(x)=1\right\}$. We say that the duality mapping $x \mapsto D(x)$ is upper semi-continuous and compact valued at $x \in S(X)$ if $D(x)$ is compact and given $\varepsilon>0$ there exists a $\delta>0$ such that $D(y) \subseteq D(x)+\varepsilon B\left(X^{*}\right)$ for all $\|x-y\|<\delta$.

Now property $(\alpha)$ at $f \in S\left(X^{*}\right)$ can be characterised by such a continuity property of the duality mapping for $X^{*}$ at $f$.

We need the following properties of slices.

Lemma 1. [5, Lemma 2.1, p.102]. Given $f \in S\left(X^{*}\right)$ and $0<\delta<1$, the slice $S\left(\bar{B}\left(X^{* *}\right), f, \delta\right)$ is contained in the weak * closure of the slice $S(\bar{B}(\hat{X}), f, \delta)$.

We will also use the following characterisation of upper semi-continuity of the duality mapping on the dual.

Lemma 2. [5, Theorem 2.2, p.102]. The duality mapping $f \mapsto D(f)$ for $X^{*}$ is upper semi-continuous at $f \in S\left(X^{*}\right)$ if and only if for given $\varepsilon>0$ there exists a $0<\delta<1$ such that $S(\bar{B}(\widehat{X}), f, \delta) \subseteq D(f)+\varepsilon B\left(X^{* *}\right)$.

THEOREM 3. The norm $\|\cdot\|$ of $X$ has property $(\alpha)$ at $f \in S\left(X^{*}\right)$ if and only if the duality mapping $f \mapsto D(f)$ for $X^{*}$ is upper semi-continuous and compact valued at $f$.

ProOF: Suppose that the duality mapping $f \mapsto D(f)$ for $X^{*}$ is upper semicontinuous at $f$. Then it follows from Lemma 2 that given $\varepsilon>0$ there exists a $0<\delta_{\varepsilon}<1$ such that

$$
S(\bar{B}(\widehat{X}), f, \delta) \subseteq D(f)+\varepsilon B\left(X^{* *}\right) \quad \text { for all } 0<\delta<\delta_{\varepsilon} .
$$

So

$$
\alpha(S(\bar{B}(\widehat{X}), f, \delta)) \leq \alpha\left(D(f)+\epsilon B\left(X^{* *}\right)\right) \quad \text { for all } 0<\delta<\delta_{\varepsilon} \text {. }
$$

But if $D(f)$ is compact then $\alpha\left(D(f)+\varepsilon B\left(X^{* *}\right)\right) \leqslant 2 \varepsilon$ which implies that the norm $\|\cdot\|$ of $X$ has property $(\alpha)$ at $f$.

Conversely, suppose that the norm $\|\cdot\|$ of $X$ has property $(\alpha)$ at $f$. Then $A \equiv \bigcap_{\delta>0} S(B(X), f, \delta)=\{x \in S(X): f(x)=1\}$ is a non-empty compact set. So 
given $\varepsilon>0$ there exists a $0<\delta<1$ such that

Then

$$
\begin{gathered}
S(\bar{B}(X), f, \delta) \subseteq A+\varepsilon B(X) . \\
S(\bar{B}(\widehat{X}), f, \delta) \subseteq D(f)+\varepsilon B\left(X^{* *}\right)
\end{gathered}
$$

which by Lemma 2 implies that the duality mapping $f \mapsto D(f)$ on $X^{*}$ is upper semicontinuous at $f$. But also

$$
S(\bar{B}(\widehat{X}), f, \delta) \subseteq \widehat{A}+\varepsilon \bar{B}\left(X^{* *}\right)
$$

As $\hat{A}$ is weak * closed and $\bar{B}\left(X^{* *}\right)$ is weak * compact then $\hat{A}+\varepsilon \bar{B}\left(X^{* *}\right)$ is weak * closed and so contains the weak * closure of $S(\bar{B}(\hat{X}), f, \delta)$. But from Lemma 1 , the weak * closure of $S(\bar{B}(\hat{X}), f, \delta)$ contains $S\left(\bar{B}\left(X^{* *}\right), f, \delta\right)$ so

$$
S\left(\bar{B}\left(X^{* *}\right), f, \delta\right) \subseteq \widehat{A}+\varepsilon \bar{B}\left(X^{* *}\right)
$$

which implies that

$$
D(f) \subseteq \widehat{A}+\varepsilon \bar{B}\left(X^{* *}\right)
$$

However, $A$ is compact so $\alpha\left(\widehat{A}+\varepsilon \bar{B}\left(X^{* *}\right)\right) \leq 2 \varepsilon$ from which we deduce that $D(f)$ is compact.

The norm $\|\cdot\|$ of $X$ is said to have the Radon-Riesz property $H$ if for any sequence $\left\{x_{n}\right\}$ in $X$ which is weakly convergent to $x$ and $\left\|x_{n}\right\| \rightarrow\|x\|$ we have that $\left\{x_{n}\right\}$ is norm convergent to $x$. Montesinos $[8$, Theorem 3, p.96] proved that the norm $\|\cdot\|$ has the drop property if and only if it has property $(\alpha)$ for all $f \in S\left(X^{*}\right)$ if and only if it has property $H$ and $X$ is reflexive.

So from Montesinos' result and Theorem 3 we can make the following deduction.

COROLlary 4. The norm $\|\cdot\|$ of $X$ has the drop property if and only if the duality mapping $f \mapsto D(f)$ for $X^{*}$ is upper semi-continuous and compact valued on $S\left(X^{*}\right)$.

A particular feature of this result is that it suggests a generalisation of the drop property which could provide a drop characterisation of reflexivity.

We will say that the norm $\|\cdot\|$ of $X$ has the weak drop property if for every weakly sequentially closed set $C$ disjoint from $\bar{B}(X)$ there exists an $x \in C$ such that $D(x, \bar{B}(X)) \cap C=\{x\}$.

If we denote by $\tau$ the weak * or weak topology on $X^{*}$ we say that the duality mapping $x \mapsto D(x)$ for $X$ is $(n-\tau)$ upper semi-continuous and $\tau$-compact valued at $x \in S(X)$ if $D(x)$ is $\tau$-compact and given a $\tau$-open neighbourhood $N$ of 0 in $X^{*}$ 
there exists a $\delta>0$ such that $D(y) \subseteq D(x)+N$ for all $\|x-y\|<\delta$. Now the duality mapping $x \mapsto D(x)$ is always $\left(n-w^{*}\right)$ upper semi-continuous and $w^{*}$-compact valued on $S(X)$.

For the weak drop property we have the following significant characterisation.

ThEOREM 5. Given a Banach space $(X,\|\cdot\|)$, the following statements are equivalent.

(i) the norm $\|\cdot\|$ has the weak drop property,

(ii) every stream in $X \backslash \bar{B}(X)$ contains a weakly convergent subsequence,

(iii) the space $X$ is reflexive,

(iv) the duality mapping $f \rightarrow D(f)$ for $X^{*}$ is $(n-w)$ upper semi-continuous and $w$-compact valued on $S\left(X^{*}\right)$.

Proof: (i) $\Rightarrow$ (ii) Suppose that there exists a stream $\left\{x_{n}\right\}$ in $X$ which does not contain a weakly convergent subsequence. Then $C \equiv\left\{n_{n}: x \in N\right\}$ is a weakly sequentially closed set. From the stream property, $x_{n+1} \in D\left(x_{n}, \bar{B}(X)\right)$ for all $n$ and we see that there is no $n$ such that $D\left(x_{n}, \bar{B}(X)\right) \cap C=\left\{x_{n}\right\}$, so $X$ does not have the weak drop property.

(ii) $\Rightarrow$ (i) Suppose that the norm $\|\cdot\|$ does not have the weak drop property. Then there exists a weakly sequentially closed set $C$ disjoint from $\bar{B}(X)$ such that for each $z \in C$

$$
\inf \{\|x\|: x \in C \cap D(z, \bar{B}(X))\}=1,
$$

otherwise we would contradict Daneš' drop theorem [3]. So we can choose a sequence $\left\{x_{n}\right\}$ in $C$ such that $x_{n+1} \in D\left(x_{n}, \bar{B}(X)\right)$ and $\lim _{n \rightarrow \forall}\left\|x_{n}\right\|=1$. But the stream $\left\{x_{n}\right\}$ has a weakly convergent subsequence $\left\{x_{n_{k}}\right\}$. Since the norm $\|\cdot\|$ is weakly lower semicontinuous, for the limit $x_{0}$ of $\left\{x_{n_{k}}\right\}$ we have $\left\|x_{0}\right\| \leq 1$. Then $x_{0} \in \bar{B}(X)$. But $C$ is also weakly sequentially closed so $x_{0} \in C$. This contradicts the fact that $\bar{B}(X)$ and $C$ are disjoint.

(ii) $\Rightarrow$ (iii) Given $f \in S\left(X^{*}\right)$ choose a sequence $\left\{y_{n}\right\}$ in $S(X)$ such that $f\left(y_{n}\right)>$ $1-1 / 4^{n}$ for each $n$. Then we inductively construct a sequence $\left\{x_{n}\right\}$ as follows. Choose $x_{1} \in 2 \bar{B}(X)$ with $f\left(x_{1}\right)>2-1 / 4$ and then

$$
x_{n+1}=\left(x_{n}+y_{n}\right) / 2 \quad \text { for each } n .
$$

We can verify by induction that for each $n$,

$$
1+\frac{3}{4^{n}} \leq f\left(x_{n}\right) \leq\left\|x_{n}\right\| \leq 1+\frac{1}{2^{n-1}}
$$

and clearly then $x_{n+1} \in D\left(x_{n}, \bar{B}(X)\right)$; that is, the sequence $\left\{x_{n}\right\}$ is a stream. But then $\left\{x_{n}\right\}$ has a weakly convergent subsequence $\left\{x_{n_{k}}\right\}$ whose limit $x_{0}$ satisfies

$$
f\left(x_{0}\right)=\lim _{k \rightarrow \forall} f x_{n_{k}}\left(x_{n_{k}}\right)=1 \text { and }\left\|x_{0}\right\| \leq 1 .
$$


So $f$ attains its norm at $x_{0} \in \bar{B}(X)$. We conclude from James' characterisation of reflexivity that $X$ is reflexive.

(iii) $\Rightarrow$ (ii) This follows directly from the Eberlein-Smulian characterisation of reflexivity $[4$, p.430].

(iii) $\Rightarrow$ (iv) This follows from the fact that the weak * topology is the weak topology for a reflexive space.

(iv) $\Rightarrow$ (iii) Suppose that (iv) holds but that $X$ is not reflexive. Then from James' characterisation of reflexivity there exists an $f \in S\left(X^{*}\right)$ which does not attain its norm on $\bar{B}(X)$. Then $D(f)$ is a weakly compact convex set disjoint from the weakly closed convex set $\bar{B}(\widehat{X})$. So $D(f)$ and $\bar{B}(\widehat{X})$ can be strongly separated by a weakly continuous linear functional on $X^{* *}$. However, the Bishop-Phelps Theorem [2] tells us that the continuous linear functionals in $S\left(X^{*}\right)$ which attain their norm on $\bar{B}(X)$ are dense in $S\left(X^{*}\right)$. These two statements contradict the duality mapping $f \mapsto D(f)$ for $X^{*}$ being $(n-w)$ upper semi-continuous at $f$.

\section{REFERENCES}

[1] J. Banas and K. Goebel, Measures of non-compactness in Banach spaces (Marcel Dekker, New York and Basel, 1980).

[2] E. Bishop and R.R. Phelps, 'A proof that every Banach space is subreflexive', Bull. Amer. Math. Soc. 67 (1961), 97-98.

[3] J. Daneš, 'A geometric theorem useful in non-linear functional analysis', Boll. Un. Mat. Ital. 6 (1972), 369-372.

[4] Nelson Dunford and Jacob T. Schwartz, Linear Operators Part I: General Theory (Wiley - Interscience, New York, 1957).

[5] J.R. Giles, D.A. Gregory and Brailey Sims, 'Geometrical implications of upper semicontinuity of the duality mapping on a Banach space', Pac. J. Math. 79 (1978), 99-109.

[6] R.C. James, 'Characterisations of reflexivity', Studia Math. 23 (1964), 205-216.

[7] D.N. Kutzarova, 'A sufficient condition for the drop property', C.R. Acad. Bulgare Sci. 39 (1986), 17-19.

[8] V. Montesinos, 'Drop property equals reflexivity', Studia Math. 87 (1987), 93-100.

[9] S. Rolewicz, 'On drop property', Studia Math. 85 (1987), 27-35.

\footnotetext{
Department of Mathematics

The University of Newcastle

Newcastle NSW 2308

Australia
} 